Proyecciones Journal of Mathematics

Vol. 32, No 4, pp. 409-418, December 2013.

Universidad Católica del Norte

Antofagasta - Chile

DOI: 10.4067/S0716-09172013000400008

\title{
Fixed points and common fixed points theorems in pseudo-ordered sets
}

\author{
Abdelkader Stouti and Abdelhakim Maaden \\ University Sultan Moulay Slimane, Morocco \\ Received: May 2011. Accepted: July 2013
}

Under suitable conditions, we establish the existence of the greatest and the least fixed points of monotone maps defined on nonempty pseudoordered sets. Also, we prove that the set of all common fixed points of two categories of finite commutative family $\mathcal{F}$ of monotone maps $f$ defined on a nonempty complete trellis is also a nonempty complete trellis.

2000 Mathematics Subject Classification : 06B23, 54C60,47H10.

Keywords and phrases : Pseudo-ordered set, fixed point, monotone map, complete trellis. 


\section{Introduction}

In 1971, H. Skala introduced the notions of pseudo-ordered sets and trellises and gave two fixed points theorems in this setting (see [Theorems 36 and 37,11$]$ ). Unfortunately this subject was a long time without serious study because in this setting we have not the essential property used in all proofs for fixed point theory in the case of partially ordered sets namely the property of transitivity (for examples see: $[3,5,12,13,15]$ ). Later on, S. Parameshwara Bhatta and all [2,3] studied the fixed point property in pseudo-ordered sets. In the present paper, we first establish the existence of the least and the greatest fixed points of monotone maps defined on nonempty pseudo-ordered sets (see Theorems 3.1 and 3.3). Note that our results ensure the existence of the least and the greatest fixed points under weaker hypothesis than the assumptions of [Theorem 36, 11] which was established by H. Skala. We prove also that the set of all common fixed points of two categories of finite commutative family $\mathcal{F}$ of monotone maps $f$ defined on a nonempty complete trellis is also a nonempty complete trellis (see Theorems 4.1 and 4.2). Note that our reslts could be used as a tool for solving differential equations in metric pseudo-ordered spaces (for the case of metric ordered spaces see for examples: $[1,2,4,6,7,15])$.

\section{Preliminaries}

Let $X$ be a nonempty set and $\unrhd$ be a binary relation defined on its. If the binary relation $\unrhd$ is reflexive and antisymmetric, we say that $(X, \unrhd)$ is a pseudo-ordered set or a psoset.

Let $A$ be a nonempty subset of a psoset $(X, \unrhd)$.

An element $u$ of $X$ is said to be an upper bound of $A$ (respectively $v$ a lower bound of $A$ ) if $x \unrhd u$ for every $x \in A$. An element $s$ of $X$ is called a greatest element or the maximum of $A$ and denoted by $s=\max (A, \unrhd)$ if $s$ is an upper bound of $A$ and $s \in A$.

An element $v$ of $X$ is said to be a lower bound of $A$ if $v \unrhd x$ for every $x \in A$. An element $\ell$ of $X$ is called a least or the minimum element of $A$ and denoted by $\ell=\min (A, \unrhd)$ if $\ell$ is a lower bound of $A$ and $\ell \in A$.

When the least upper bound (l.u.b.) $s$ of $A$ exists, we shall denoted its by $s=\sup (A, \unrhd)$. Dually if the greatest lower bound (g.l.b.) of $A$ exists, we shall denoted its by $\ell=\inf (A, \unrhd)$. 
Note that the greatest lower bound and the least upper bound when they exist they are unique.

A psoset $(X, \unrhd)$ is said to be a trellis if every pair of elements of $(X, \unrhd)$ has a greatest lower bound (g.l.b) and a least upper bound (l.u.b). A psoset $(X, \unrhd)$ is said to be a complete trellis if every nonempty subset of $X$ has a g.l.b and a l.u.b. For more details for these notions can be found in H.L. Skala $[10,11]$.

Let $(X, \unrhd)$ be a nonempty pseudo-ordered sets and let $f: X \rightarrow X$ be a map. We shall say that $f$ is monotone if for every $x, y \in X$, with $x \unrhd y$, then we have $f(x) \unrhd f(y)$.

An element $x$ of $X$ is said to be a fixed point of a map $f: X \rightarrow X$ if $f(x)=x$. The set of all fixed points of $f$ is denoted by Fix $(f)$.

\section{Example.}

Let $A$ the set defined by $A=\{0, a, b, c\}$. We define a pseudo-order relation on $A$ by setting:

(i) for every $x \in A$, we have $0 \unrhd x$ and

(ii) $a \unrhd b \unrhd c \unrhd a$.

Then, $(A, \unrhd)$ is a trellis having the minimum element 0 but $(A, \unrhd)$ is not complete.

In this paper, we shall need the following notion of inverse relation.

Definition 2.1. Let $X$ be a nonempty set and let $\unrhd$ be a relation on its. The inverse relation $\unlhd$ of $\unrhd$ is defined for every $x, y \in X$ by:

$$
(x \unlhd y) \Leftrightarrow(y \unrhd x) .
$$

The proofs of the following two lemmas are obvious.

Lemma 2.2. Let $\unrhd$ be a pseudo-order relation defined on a nonempty set $X$ and let $\unlhd$ be the inverse relation of $\unrhd$. Then, $\unlhd$ is a pseudo-order relation on $X$.

Lemma 2.3. Let $\unrhd$ be a pseudo-order relation defined on a nonempty set $X$, let $\unlhd$ be the inverse relation of $\unrhd$ and let $A$ be a nonempty subset of $X$. Then, we have

(i) if $\sup (A, \unrhd)$ exists, $\operatorname{so} \inf (A, \unlhd)$ exists too and $\sup (A, \unrhd)=\inf (A, \unlhd)$ 
(ii) if $\inf (A, \unrhd)$ exists, hence also $\sup (A, \unlhd)$ exists too and $\inf (A, \unrhd)=$ $\sup (A, \unlhd)$;

(iii) if $\min (A, \unrhd)$ exists, then also $\max (A, \unlhd)$ exists and $\min (A, \unrhd)=$ $\max (A, \unlhd)$;

(iv) if $\max (A, \unrhd)$ exists, so also $\min (A, \unlhd)$ exists too and $\max (A, \unrhd)=$ $\min (A, \unlhd)$;

$(\mathrm{v})$ if $(X, \unrhd)$ is a nonempty complete trellis, so $(X, \unlhd)$ is also a nonempty complete trellis ;

(vi) if $f: X \rightarrow X$ is a monotone map for $\unrhd$, then $f$ is also a monotone map for $\unlhd$.

\section{Fixed points in pseudo-ordered sets}

In this section we shall establish the existence of the greatest and the least fixed points of monotone maps defined on a nonempty pseudo-ordered sets. Note that our results (Theorem 3.1 and 3.3) ensure the existence of the least and the greatest fixed points under weaker hypothesis than the assumptions of [Theorem 36, 11] which was established by H. Skala. First, we shall give our key result in this paper.

Theorem 3.1. Let $(X, \unrhd)$ be a nonempty pseudo-ordered set with a least element $\ell$. Assume that every nonempty subset of $X$ has a supremum in $(X, \unrhd)$. Then, the set of all fixed points of every monotone map $f$ : $(X, \unrhd) \rightarrow(X, \unrhd)$ is nonempty and has a least element.

Proof. Let $(X, \unrhd)$ be a nonempty pseudo-ordered set with a least element $\ell$ and $f:(X, \unrhd) \rightarrow(X, \unrhd)$ be a monotone map.

First step. We have: $F i x(f) \neq \emptyset$. Indeed, let $\mathcal{A}$ the family of all subsets $A$ of $X$ satisfying the following three conditions:

(i) $\ell \in A$;

(ii) $f(A) \subset A$;

(iii) for every nonempty subset $B$ of $A$, we have $\sup (B, \unrhd) \in A$.

Let us note that $\mathcal{A} \neq \emptyset$ because $X \in \mathcal{A}$. Now, let $C=\bigcap_{A \in \mathcal{A}} A$.

Claim 1. $C$ is the nonempty least element of $\mathcal{A}$. Indeed, as $\ell \in A$ for every $A \in \mathcal{A}$, so $\ell \in C$. Since $C=\bigcap_{A \in \mathcal{A}} A$, then we have

$$
f(C)=f\left(\bigcap_{A \in \mathcal{A}} A\right) \subset \bigcap_{A \in \mathcal{A}} f(A) \subset \bigcap_{A \in \mathcal{A}} A .
$$


Thus, we get $f(C) \subset C$. Now, let $D \subset C$ such that $D \neq \emptyset$. Then, $D \subset A$ for every $A \in \mathcal{A}$. So, $\sup (D, \unrhd) \in A$ for every $A \in \mathcal{A}$. Hence, we obtain $\sup (D, \unrhd) \in C$. Therefore, $C$ is the least nonempty element of $\mathcal{A}$. Then, we set $s=\sup (C, \unrhd)$.

Claim 2. We have: $s \in F i x(f)$. Indeed, since $s \in C$ and $f(C) \subset C$, then $f(s) \in C$. As $s=\sup (C, \unrhd)$, so we get

$$
f(s) \unrhd s .
$$

Now, we consider the following subset $D$ of $C$ defined by

$$
D=\{x \in C: x \unrhd f(s)\} .
$$

As $\ell \in D$, so $D \neq \emptyset$. We claim that $D \in \mathcal{A}$. As $D \subset C$, then we get $x \unrhd s$ for every $x \in D$. By the monotonicity of $f$ we obtain $f(x) \unrhd f(s)$, for every $x \in D$. Then, we get $f(D) \subset D$.

Now, if $E \subset D$ and $E \neq \emptyset$, then we set $m=\sup (E, \unrhd)$. As $E \subset D$, so $E \subset C$ and $m \in C$. By our definition of $D$, we deduce that $f(s)$ is an upper bound of $(E, \unrhd)$. Then, we get $m \unrhd f(s)$. Thus, we have $m \in D$. Therefore, we obtain $D \in \mathcal{A}$. As $D \subset C$ and $C$ is the least nonempty element of $\mathcal{A}$, so we get $C=D$. On the other hand, we know that $s=\sup (C, \unrhd) \in C$, then we obtain

$$
s \unrhd f(s) .
$$

By combining (2.1) and (2.2) and using the antisymmetry of $\unrhd$, we obtain that $f(s)=s$. Thus, we have $s \in F i x(f)$. Therefore, Fix $(f) \neq \emptyset$.

Second step. We have: $(F i x(f), \unrhd)$ has a least element. Indeed, from the first step above, we know that $F i x(f) \neq \emptyset$. Next, we shall show that $(F i x(f), \unrhd)$ has a least element. Now we consider the following subset $S$ of $X$ defined by

$$
S=\{x \in X: x \unrhd z \text { for every } z \in F i x(f)\} .
$$

As $\ell=\min (X, \unrhd)$, then $\ell \in S$. Hence, $\ell=\min (S, \unlhd)$. Now let $L$ be a nonempty subset of $S$. Hence, by our hypothesis $L$ has a supremum in $(X, \unlhd)$. Let $t=\sup (L, \unrhd)$. As $L \subset S$, so every element $z$ of $F i x(f)$ is an upper bound of $(L, \unrhd)$. Since $t=\sup (L, \unrhd)$, hence we get $t \unrhd z$ for every $z \in$ Fix $(f)$. Then, we have $t \in S$. So, $\sup (L, \unrhd) \in S$. Hence, $\sup (L, \unrhd)$ is the supremum of $L$ in $(S, \unrhd)$. 
Now, let $x \in S$. So by our definition of $S$, we have $x \unrhd z$ for every $z \in$ Fix $(f)$. Hence, by the monotonicity of $f$ we get $f(x) \unrhd z$ for every $z \in$ Fix $(f)$. Then, $f(x) \in S$ for every $x \in S$. Thus, we have $f(S) \subset S$.

Therefore, all hypothesis of the first step above are satisfied for the map $f_{/ S}:(S, \unrhd) \rightarrow(S, \unrhd)$ defined by $f_{/ S}(x)=f(x)$ for every $x \in S$. Then, we get $F i x\left(f_{/ S}\right) \neq \emptyset$. So, there exists $a \in S$ such that $f(a)=a$ and $a \unrhd z$ for every $z \in F i x(f)$. Thus, $a \in F i x(f)$ and $a$ is a lower bound of $(F i x(f), \unrhd)$. Thus, $a$ is the minimum of $(F i x(f), \unrhd)$.

As a corollary of Theorem 3.1, we get the following result.

Corollary 3.2. Let $(X, \leq)$ be a nonempty partially ordered set with a least element $\ell$. Assume that every nonempty subset of $X$ has a supremum in $(X, \leq)$. Then, the set of all fixed points of every monotone map $f:(X, \leq$ )$\rightarrow(X, \leq)$ is nonempty and has a least element.

Next, by using Theorem 3.1 and Lemmas 2.2 and 2.3, we obtain the following result.

Theorem 3.3. Let $(X, \unrhd)$ be a nonempty pseudo-ordered set with a greatest element $g$. Assume that every nonempty subset of $X$ has an infimum in $(X, \unrhd)$. Then, the set of all fixed points of every monotone map $f$ : $(X, \unrhd) \rightarrow(X, \unrhd)$ is nonempty and has a greatest element.

Proof. Let $(X, \unrhd)$ be a nonempty pseudo-ordered set with a greatest element $g$, let $f:(X, \unrhd) \rightarrow(X, \unrhd)$ be a monotone map and let $\unlhd$ be the inverse relation of $\unrhd$. From Lemma 2.2 , we know that $\unlhd$ is a pseudo-order relation on $X$. On the other hand, by Lemma $2.3, \min (X, \unlhd)$ exists and we have $\min (X, \unlhd)=g$. As by our hypothesis $f:(X, \unrhd) \rightarrow(X, \unrhd)$ is a monotone map, so from Lemma 2.3 the map $f:(X, \unlhd) \rightarrow(X, \unlhd)$ is also monotone. Thus, all hypothesis of Theorem 3.1 are satisfied. Therefore, The set Fix $(f)$ of all fixed points of the map $f$ is nonempty and has a least element in $(X, \unlhd), m$, say. Then from Lemma 2.3 , we get $m=\min (\operatorname{Fix}(f), \unlhd)=\max (\operatorname{Fix}(f), \unrhd)$.

As a consequence of Theorem 3.3, we get the following result.

Corollary 3.4. Let $(X, \leq)$ be a nonempty partially ordered set with a greatest element $g$. Assume that every nonempty subset of $X$ has an infimum in $(X, \leq)$. Then, the set of all fixed point of every monotone map $f:(X, \leq) \rightarrow(X, \leq)$ is nonempty and has a greatest element. 
Combining Theorems 3.1 and 3.3, we obtain the following results.

Corollary 3.5. Let $(X, \unrhd)$ be a nonempty complete trellis. Then, the set of all fixed point of every monotone map $f:(X, \unrhd) \rightarrow(X, \unrhd)$ is nonempty and has a greatest and a least elements.

Corollary 3.6. Let $(X, \leq)$ be a nonempty complete lattice. Then, the set of all fixed points of every monotone map $f:(X, \leq) \rightarrow(X, \leq)$ is nonempty and has a least and a greatest elements.

\section{Common fixed points in compete trellises}

In this section, we shall prove under suitable conditions that the set of all common fixed points of a finite commutative family $\mathcal{F}$ of monotone maps $f$ defined on a nonempty complete trellis is also a nonempty complete trellis. First, we shall show the following.

Theorem 4.1. Let $(X, \unrhd)$ be a nonempty complete trellis and $\mathcal{F}$ be a finite commutative family of monotone maps $f:(X, \unrhd) \rightarrow(X, \unrhd)$ such that for every $x \in X$, and $f \in \mathcal{F}$ we have $x \unrhd f(x)$. Then, the set of all common fixed points $\mathrm{Fix}(\mathcal{F})$ of $\mathcal{F}$ is a nonempty complete trellis.

Proof. Let $(X, \unrhd)$ be a nonempty complete trellis, let $\mathcal{F}=\left\{f_{1}, \ldots, f_{n}\right\}$ be a finite commutative family of monotone maps $f_{i}:(X, \unrhd) \rightarrow(X, \unrhd)$ for $i=1, \ldots, n$ (i.e. $f_{i} \circ f_{j}=f_{j} \circ f_{i}$ for every $i, j \in\{1, \ldots, n\}$ ). and let $g=\max (X, \unrhd)$. Then, $f_{i}(g)=g$ for every $i=1, \ldots, n$. Hence, $\operatorname{Fix}(\mathcal{F})$ is nonempty and has a greatest element. From [Theorem 37, 5], we know that Fix $\left(f_{1}\right)$ is a nonempty complete trellis. Now, we shall show that the set of common fixed points of the family $\left\{f_{1}, f_{2}\right\}$ is a nonempty complete trellis. Indeed, if $x \in F i x\left(f_{1}\right)$, then $f_{2}(x)=f_{2}\left(f_{1}(x)\right)=f_{1}\left(f_{2}(x)\right)$. So, for every $x \in \operatorname{Fix}\left(f_{1}\right)$ we have $f_{2}(x) \in \operatorname{Fix}\left(f_{1}\right)$. So, $f_{2}\left(F i x\left(f_{1}\right)\right) \subset F i x\left(f_{1}\right)$. Then as Fix $\left(f_{1}\right)$ is a nonempty complete trellis and $x \unrhd f_{2}(x)$ for every $x \in$ Fix $\left(f_{1}\right)$, hence from [Theorem 37,5] the set of all fixed points of $f_{2}$ in Fix $\left(f_{1}\right)$ is a nonempty complete trellis. On the other hand, the set of all fixed points of $f_{2}$ in $F i x\left(f_{1}\right)$ is equal to $F i x\left(\left\{f_{1}, f_{2}\right\}\right)$. Thus, the set of common fixed points of the family $\left\{f_{1}, f_{2}\right\}$ is a nonempty complete trellis. By induction, assume that for every $k \in\{1, \ldots, n-1\}$ the set of all common fixed points of the family $\left\{f_{1}, \ldots, f_{k}\right\}$ is a nonempty complete trellis. Let $x \in \operatorname{Fix}\left(\left\{f_{1}, \ldots, f_{n-1}\right\}\right)$. Then, $f_{n}(x)=f_{n}\left(f_{k}(x)\right)=f_{k}\left(f_{n}(x)\right)$ for every $k \in\{1, \ldots, n-1\}$. So, $f_{n}(x) \in F i x\left(f_{k}\right)$ for every $k \in\{1, \ldots, n-1\}$. 
Thus, $f_{n}\left(F i x\left(\left\{f_{1}, \ldots, f_{n-1}\right\}\right)\right) \subset F i x\left(\left\{f_{1}, \ldots, f_{n-1}\right\}\right)$. As by the hypothesis of induction $F i x\left(\left\{f_{1}, \ldots, f_{n-1}\right\}\right)$ is a nonempty complete trellis and $x \unrhd f_{n}(x)$ for every $x \in \operatorname{Fix}\left(\left\{f_{1}, \ldots, f_{n-1}\right\}\right)$, then we deduce from [Theorem 37, 5] that the set $F i x\left(\left\{f_{1}, \ldots, f_{n}\right\}\right)=\bigcap_{i=1}^{i=n} F i x\left(f_{i}\right)$ is a nonempty complete trellis.

Next, by using Theorem 4.1 and Lemmas 2.2 and 2.3, we obtain the following dual result.

Theorem 4.2. Let $(X, \unrhd)$ be a nonempty complete trellis and $\mathcal{F}$ a finite commutative family of monotone map $f:(X, \unrhd) \rightarrow(X, \unrhd)$ such that for every $x \in X$, and $f \in \mathcal{F}$ we have $f(x) \unrhd x$. Then, the set of all common fixed points $\operatorname{Fix}(\mathcal{F})$ of $\mathcal{F}$ is a nonempty complete trellis.

Proof. Let $(X, \unrhd)$ be a nonempty complete trellis and $\mathcal{F}$ a finite commutative family of monotone map $f:(X, \unrhd) \rightarrow(X, \unrhd)$ such that for every $x \in X$, and $f \in \mathcal{F}$ we have $f(x) \unrhd x$ and let $\unlhd$ be the inverse relation of $\unrhd$. From Lemma 2.2, we know that $\unlhd$ is a pseudo-order relation on $X$. On the other hand, by Lemma 2.3 the pseudo-ordered set $(X, \unlhd)$ is a nonempty complete trellis. Let $f \in \mathcal{F}$. As by our hypothesis $f:(X, \unrhd) \rightarrow(X, \unrhd)$ is a monotone map, so from Lemma 2.3 the map $f:(X, \unlhd) \rightarrow(X, \unlhd)$ is also monotone. Since $f(x) \unrhd x$ for every $x \in X$ and $f \in \mathcal{F}$, so we get $x \unlhd f(x)$ for every $x \in X$ and $f \in \mathcal{F}$. Therefore from Theorem 3.1, we know that the set $\left(F i x\left(\left\{f_{1}, \ldots, f_{n}\right\}\right), \unlhd\right)$ is a nonempty complete trellis. Hence by Lemma 2.3 , we deduce that $\left(\operatorname{Fix}\left(\left\{f_{1}, \ldots, f_{n}\right\}\right), \unrhd\right)$ is a nonempty complete trellis.

\section{References}

[1] Agarwal, R. P., El-Gebeily, M. A., and ORegan, D. : Generalized contractions in partially ordered metric spaces, Applicable Analysis, vol. 87, no. 1, pp. 109116, (2008).

[2] Altun I., Simsek H.: Some fixed point theorems on ordered metric spaces and application. Fixed Point Theory Appl 2010 ; 2010. Article ID 621469, 17 pages, doi:10.1155/2010/621469.

[3] Amann, H., Order structures and fixed points, Ruhr-universitãt, Bochum, mimeographed lecture notes, (1977). 
[4] Hemant Kumar Nashine, Bessem Samet and Calogero Vetro (2012): Fixed Point Theorems in Partially Ordered Metric Spaces and Existence Results for Integral Equations, Numerical Functional Analysis and Optimization, 33:11, pp. 1304-1320

[5] Knaster, B.: un théorème sur les fonctions d'ensembles, Annales de la Societé Polonaise de Mathématique vol. 6, pp. 133-134, (1968).

[6] Nieto, J. J. and Rodríguez-Lopez, R.: Contractive mapping theorems in partially ordered sets and applications to ordinary differential equations, Order, vol. 22, no. 3, pp. 223239, (2005).

[7] Nieto, J. J. and Rodríguez-Lopez, R.: Existence and uniqueness of fixed point in partially ordered sets and applications to ordinary differential equations, Acta Mathematica Sinica, vol. 23, no. 12, pp. 22052212, (2007).

[8] Parameshwara Bhatta, S., Shashirekha, H.: A characterisation of completeness for Trellises. Algebra univeralis 44, pp. 305-308, (2000).

[9] Parameshwara Bhatta, S.: Weak chain completeness and fixed point property for pseudo-ordered sets. Czechoslovac Mathematical Journal 55 (130), pp. 365-369, (2005)

[10] Skala, H. L.: Trellis theory. Algebra Universalis 1, pp. 218-233, (1971)

[11] Skala, H. L.: Trellis theory. Mem. Amer. Math. Soc. 121, Providence, (1972)

[12] Stouti, A. : A generalized Amman fixed point theorem and its applications to Nash equilibrium, Acta Academiae Paedagogicae Ny'iregyh'aziensis, 21, no. 2, pp. 107-112, (2005).

[13] Tarski, A.: A Lattice-Theoretical Fixpoint Theorem and its Applications Pacific J. Math, pp. 285-309 5(1955)

[14] Ran, A. C. M. and Reurings, M. C. B.: A fixed point theorem in partially ordered sets and some applications to matrix equations, Proceedings of the American Mathematical Society, vol. 132, no. 5, pp. 14351443, (2004).

[15] E. Zeidler: Nonlinear Functional Analysis and its Applications, I. Fixed-Point Theorems, Springer-Verlag, New-York, (1986). 


\title{
Abdelkader Stouti
}

Center for Doctoral Studies: Sciences and Techniques, Laboratory of Mathematics and Applications, Faculty of Sciences and Techniques, University Sultan Moulay Slimane, P. O. Box 523. Beni-Mellal 23000, Morocco

e-mail : stouti@yahoo.com or stout@fstbm.ac.ma

and

\author{
Abdelhakim Maaden \\ Center for Doctoral Studies: Sciences and Techniques, \\ Laboratory of Mathematics and Applications, \\ Faculty of Sciences and Techniques, \\ University Sultan Moulay Slimane, \\ P. O. Box 523. Beni-Mellal 23000, \\ Morocco
}

